\title{
Análisis crítico de los conceptos de sostenibilidad y desarrollo sostenible
}

\author{
M.Sc. Rose Marie Menacho Odio \\ Bióloga. Tutora e investigadora, Cátedra Ecología y Educación Ambiental, Escuela de Ciencias Exactas y Naturales, \\ Universidad Estatal a Distancia. Costa Rica; rmenacho@uned.ac.cr \\ Apdo. 572-1250, Escazú, San José, Costa Rica. Tel. 88379384.
}

Recibido: 17 abril 2013

Aceptado: 05 junio 2013

\section{RESUMEN}

Se analiza y critica el concepto de "Desarrollo Sostenible" por ser un término que no ha logrado producir una visión unificada del futuro, y que, por su ambigüedad, facilita que distintos grupos humanos le atribuyan significados distintos de acuerdo a la visión de desarrollo que defiendan. Estos diferentes significados parten del valor que cada grupo asigna al sistema económico, al conocimiento tradicional, la ciencia y la tecnología así como al papel que visualizan corresponde al ser humano frente a la naturaleza. Para lograr construir una visión común de futuro y un modelo sostenible, es necesario compartir valores como respeto, solidaridad, responsabilidad y colaboración hacia la naturaleza y los demás seres humanos. Para ello, la educación ambiental es un medio valioso que permita permear estos valores en la sociedad actual.

Palabras clave: Desarrollo sostenible, economía, ecología, transformación, educación ambiental.

\begin{abstract}
"Sustainable Development" concept was analyzed and criticized as a term that has failed to produce a unified vision of the future. Due to its ambiguity it facilitates that different human groups attribute different meanings according to their vision of development. These different meanings are based on the value that each group assign to the economic system, the traditional knowledge, science and technology and the role that corresponds to human on Nature. In order to build a common vision and a sustainable model, it is necessary to share values such as respect, solidarity, responsibility and collaboration towards nature and other human beings. For this reason, environmental education is a valuable as a mean to enable these values permeate our society.
\end{abstract}

Key words: Sustainable development, economics, ecology, transformation, environmental education.

Por mucho tiempo, y aún en la actualidad, muchas personas han considerado el desarrollo como el estado deseable para un país o región, donde se llegan a satisfacer las necesidades humanas, se cuenta con abundancia de recursos y se han solucionado problemas como el hambre, falta de empleo y la pobreza.

Para alcanzar dicho estado de "progreso", en las últimas décadas se ha venido apostado por el crecimiento económico, basado en la fe en los alcances de la ciencia, el uso de la tecnología y en la explotación de recursos naturales.

Contrario a lo esperado, junto al crecimiento económico y el supuesto alcance del nivel de "desarrollo" de algunos países, se ha dado en el mundo un aumento en los problemas ambientales y sociales. Entre los primeros vale la pena mencionar al cambio climático que amenaza con causar catástrofes de todo tipo en un futuro no muy lejano. Estos problemas, la aceptación de la limitación en la cantidad de recursos que puede producir la Tierra, un panorama de desigualdad en la distribución de la riqueza y la preocupación por el futuro de la humanidad, han impulsado la búsqueda de alternativas de modelos de desarrollo.

Un primer término formulado fue el de "Ecodesarrollo", el cual entre sus objetivos tiene la distribución equitativa del "tener", la aceptación voluntaria de las limitaciones ecológicas, el principio de solidaridad intergeneracional, la solidaridad intrageneracional, el uso de tecnologías ambientalmente apropiadas, un mayor control democrático y la participación popular en las decisiones" (Riechmann, 1995). Sin embargo, este término quedó eclipsado ante la aparición del "Desarrollo sostenible". 
En 1987, el informe de Brundtland denominado "Nuestro futuro común" introdujo el tema del futuro sostenible, y la necesidad de integrar el medio ambiente y el desarrollo (Miranda, Cruz, Machado, \& Campos, 2007). Dicho informe señala que "el desarrollo sostenible es aquel que satisface las necesidades de las generaciones actuales sin comprometer la capacidad de las generaciones futuras para satisfacer sus propias necesidades" (Riechmann, 1995). La sostenibilidad se entiende como la cualidad de poder mantenerse por sí mismo, sin ayuda exterior y sin agotar los recursos disponibles.

El concepto de desarrollo sostenible es un concepto normativo o ético-moral, pues no depende tanto de la capacidad de la madre Tierra (aunque se quieran ampliar las límites por medio de la tecnología) como del ansia de consumo de los seres humanos, y en estos recae finalmente el asumir la prescripción ética que nos impulsa a respetar estos límites (Arribas Herguedas, 2007, Riechmann, 1995). El objetivo deseable del desarrollo sostenible es no dañar la capacidad global de sustentación del planeta (Riechmann, 1995).

\section{¿Qué se le puede criticar al término de desarrollo sostenible?}

Luego de más de veinte años de acuñado el término y aunque en general haya ha sido aceptado por muchas personas, se pueden criticar varios aspectos del desarrollo sostenible:

1. Se le considera un aximoron, es decir, está compuesto por dos palabras que aparentemente tienen dos significados opuestos (Arribas Herguedas, 2007). Esto porque el desarrollo a menudo se le concibe como crecimiento económico y la sostenibilidad implica la capacidad de utilizar recursos indefinidamente. También se dice que tiene una raíz polisémica, es decir que tiene varios significados, lo cual le posibilita presentar ideas contrapuestas (Elizalbe Elvia, 2009). De hecho, los distintos significados que se le da al término desarrollo sostenible, provoca distintas respuestas (Hopwood, Mellow, \& O’Brien, 2005, Byrch, Kearins,
Milne, \& Morgan, 2009). Lo cierto es que la falta de una definición común no ayuda en nada a que se produzca un cambio, pues cada uno de los grupos está trabajando bajo su propia concepción de desarrollo, el cual a su vez refleja los valores de su grupo; además, los empresarios y similares, son los que menos están dispuestos a discutir su propia concepción de desarrollo sostenible, sin embargo, este tipo de debates es necesario si queremos alcanzar un acuerdo entre los distintos grupos (Byrch, Kearins, Milne, \& Morgan, 2009).

2. Es antropocéntrico, pues no hace mención alguna de los demás seres vivos que hay en la Tierra. Por tanto, es irreal pues no se percibe la dependencia del ser humano tanto de la naturaleza como de otros seres humanos.

3. Es bastante vago o difuso, no tiene indicadores claros para definir límites entre lo que es sostenible y lo insostenible. Aparentemente, el concepto de desarrollo sostenible nos debería de fijar límites, pero estos son tan difusos que en lugar de regular el uso de los recursos nos da luz verde para aprovecharlos, eso sí, bajo una engañosa luz de "sostenibilidad".

4. Da pie a preguntas como: ¿Cómo podemos pretender tener responsabilidades éticas hacia seres que no existen y cuyas necesidades desconocemos, si ni siquiera de las necesidades delas personas con las que en este momento cohabitamos la Tierra? ¿Qué ocurre con los demás seres del planeta? ¿No tenemos también responsabilidad hacia ellos?

\section{¿Qué se puede elogiar del desarrollo sostenible?}

El hecho de que el desarrollo sostenible haya despertado tanto interés y que, aparentemente sea apoyado por distintos grupos, brinda la oportunidad de valorar las relaciones entre humanos y naturaleza y de los humanos entre sî (Hopwood, Mellow, \& O’Brien, 2005). Esto, en teoría, debería de posibilitar intentos por llegar a acuerdos comunes. 


\section{¿Qué diferentes visiones se tienen de lo que es un desarrollo sostenible?}

Como se mencionó antes, al desarrollo sostenible se le dan muy diferentes significados. Tanto Hopwood, Mellow, \& O’Brien (2005) como Byrch, Kearins, Milne, \& Morgan (2009) han creado categorías de acuerdo a lo que las personas comprenden por este término. Este es un resumen de las distintas categorías bajo las cuales se agrupan las personas de acuerdo a lo que entienden por desarrollo sostenible:

1. Status quo: Estas personas aunque reconocen la necesidad de cambiar, siguen pensando que el desarrollo económico conduce a la sostenibilidad, y que a través del conocimiento tecnocientífico y de la empresa van a solucionar todos los problemas (Hopwood, Mellow, \& O’Brien, 2005). La visión prometeica implica que la inventiva humana, producto del desarrollo sostenido posibilitará encontrar solución a todos los problemas. Además, esta visión prometeica señala a la pobreza como la fuente de la destrucción ecológica y al continuo crecimiento económico la solución a todos de los problemas (Arribas Herguedas, 2007).

2. Reforma (Ecologismo suave). Este grupo acepta que existen problemas grandes, critican las políticas de muchos negocios y gobiernos, pero no consideran que un cambio fundamental sea necesario (Hopwood, Mellow, \& O’Brien, 2005). Se combate la contaminación y el agotamiento de los recursos naturales, pero sin hacerse cargo de las causas políticas, sociales, económicas o culturales de la crisis ambiental (Elizalbe Elvia, 2009), se concentran en cambio en las soluciones técnicas para continuar con ese modo de vida. Un ejemplo de lo que implica el punto de vista reformista son los principios operativos del desarrollo sostenible, señalados en Riechmann (1995), por ejemplo, el reducir a cero las intervenciones acumulativas, que la tasa de vaciado sea igual a la tasa de creación de sustitutos renovables, que la tasa de emisión de residuos sea igual a la tasa de asimilación de los ecosistemas y principio de selección sostenible de las tecnologías entre otros. De acuerdo a Hopwood, Mellow, \& O’Brien (2005) este punto de vista es compartido por algunas agencias gubernamentales, por expertos de ONGs y mayormente por académicos, pues se enfocan en tecnología, ciencia y en la información, con el fin de modificar el mercado y al gobierno, ya que se considera que las nuevas tecnologías van a proveer mayores beneficios económicos y sociales para la humanidad, además de proteger el ambiente. Todo lo anterior, implica cambios leves del modelo, no un modelo distinto al capitalista.

3. Ecologismo duro. Este grupo ve los problemas económicos y sociales como una consecuencia de las características de la sociedad actual y como los seres humanos nos relacionamos con el ambiente (Hopwood, Mellow, \& O’Brien, 2005). Donde el ser humano ya no se ve a sí mismo como el centro de la creación, su visión es más bien de igualdad biosférica, donde hay igualdad de derecho a vivir y prosperar a otras especies) (Elizalbe Elvia, 2009).

Hay ecologistas duros que consideran más importante al ambiente que al ser humano y piensan que una disminución radical de la población humana sería muy deseable (Hopwood, Mellow, \& O’Brien, 2005)

4. Transformistas. Son quienes consideran que el bienestar de la naturaleza y de la humanidad están íntimamente ligados, y que no es posible la una sin la otra. Esto requiere un cambio radical, por lo que son movimientos que buscan vivir sin desigualdad ni pobreza. Estas personas buscan transformar la sociedad y muchos de estos movimientos provienen del Sur del planeta (Hopwood, Mellow, \& O’Brien, 2005). Se busca la democracia, se ven las dimensiones internacionales del problema y se buscan nuevas alianzas para alcanzar una democracia real. Esta opción implica un cambio de valores, donde la naturaleza se trate con respeto, se vuelva a la ética en la convivencia humana, con el fin de vivir en unidad tanto en la diversidad 
ecológica, como en la diversidad cultural (Elizalbe Elvia, 2009).

Pienso que las diferentes visiones de desarrollo sostenible no deberían de verse como estados estáticos, sino que podrían ser manifestaciones de que el cambio hacia un nuevo paradigma ya ha iniciado.

\section{¿Cómo se debe de dar el cambio?}

Es importante percatarnos de que los distintos significados que se da al término desarrollo sostenible, surgen del valor que cada grupo de personas asigne al sistema económico, al conocimiento tradicional, la ciencia y la tecnología y el consumismo (Byrch, Kearins, Milne, \& Morgan, 2009). Estos valores tienen sus raíces de acuerdo a la creencia acerca del papel que corresponde al ser humano frente a la naturaleza.

Por ejemplo, si se considera al ser humano como un ser superior a los demás, se aceptará que se tiene derecho a dominar y a subyugar a la naturaleza; los valores como respeto, empatía y solidaridad estarán presentes, quizás hacia otras personas, pero no hacia otros seres vivos y por tanto, no se concederá que los mismos tengan derecho a bienestar, alimento, refugio y a un ambiente sano, lo mismo que el ser humano. Por tanto, el aceptar que el ser humano depende del ambiente, es insuficiente si no se tienen valores hacia el mismo como responsabilidad, respeto y compasión.

Elizalbe Elvia, (2009) plantea que el cambio debe de iniciar a un cambio a nivel personal, luego local y finalmente global. Yo considero que la educación es el principal medio para permear de valores ambientales a la sociedad. El cómo lograrlo implica un proceso de acercamiento a la naturaleza, educación que dé el valor que corresponde al agua como elemento vital, a los bosques, animales, aire y sol para retomar el espacio que el ser humano tenía en la naturaleza y del cual ahora cree haberse independizado.

La educación debe de darse en forma integral, no basarse en solo información, sino también lograr experiencias reales en la naturaleza, valerse del disfrute que despiertan los olores y colores de los bosques, permitir que las emociones formen parte de la educación. Esto implica permitir que despierte el entusiasmo y la sorpresa del descubrimiento, retomar el tiempo para observar lentamente lo que ocurre a nuestro alrededor. De igual forma, valorar la riqueza de las culturas indígenas, quienes conocen de cerca la naturaleza. Todo esto implica reinventar la educación o al menos, desarrollarla de una manera muy diferente a la actual (Orr, 2004).

\section{Conclusiones}

Como concepto, el desarrollo sostenible no ha logrado producir una visión unificada del futuro que sea aceptada por los distintos grupos de personas. Sin embargo, es necesario llegar a un entendimiento común pues la diversidad de definiciones no ayuda en nada a llegar a un acuerdo del mundo que deseamos en el futuro.

Actualmente existen grupos de personas que están proponiendo cambios en el tipo de desarrollo deseado, y que promueven la democracia, el bienestar y una nueva bioética.

Para lograr construir una visión común de futuro y un modelo sostenible, es necesario primero compartir valores como respeto, solidaridad, responsabilidad y colaboración hacia la naturaleza y los demás seres humanos.

La educación puede ser un medio para permear de esos valores a la sociedad actual, sin embargo, requiere de profundos cambios, incluyendo un acercamiento a experiencias vivenciales y a la integración de disciplinas.

\section{Trabajos citados}

Arribas Herguedas, F. (2007). La idea de desarrollo sostenible. Sistema, 75-86.

Byrch, C., Kearins, K., Milne, M., \& Morgan, R. (2009). Sustainable development: What does really mean? University of Aukland Business Review, 2-7.

Elizalbe Elvia, A. (2009). ¿Qué desarrollo puede llamarse sostenible en el siglo XXI? La cuestión de los límites y las necesidades humanas. Revista de Educación, 53-75. 
Hopwood, B., Mellow, M., \& O’Brien, G. (2005). Sustainable development: mapping different approaches. Sustainable Development, 38-52.

Miranda, T., Cruz, A., Machado, H., \& Campos, M. (2007). El desarrollo sostenible. Perspectivas y enfoques en una nueva época. Pastos y forrages, 191-204.

Orr, D. (2004). Earth in mind: On education, environment and human prospect. Washington: Island Press.
Redclift, M. (1996). Desarrollo sostenible: ampliación del alcance del debate. Recuperado el 6 de marzo de 2013, de CLADES Centro Latinoamericano de Desarrollo Sustentable: http://www.clades.cl/revistas/10/rev10per1.htm

Riechmann, J. (1995). Desarrollo sostenible: la lucha por la interpretación. En J. M. Jorge Riechmann, De la economía a la ecología (págs. 1-20). Madrid: Trotta. 
